UNIVERSIDADE DE BRASÍLIA

FACULDADE DE CIÊNCIAS DA SAÚDE

PROGRAMA DE PÓS-GRADUAÇÃO EM CIÊNCIAS DA SAÚDE

SARA KATERINE VIEIRA

EFEITO PREEMPTIVO DO IBUPROFENO NA DOR PÓSOPERATÓRIA ASSOCIADA À EXODONTIA DE TERCEIRO MOLAR: ENSAIO CLÍNICO RANDOMIZADO DUPLO-CEGO CRUZADO COMPARANDO DOSES MÚLTIPLAS VERSUS DOSE ÚNICA

\begin{abstract}
Dissertação apresentada como requisito parcial para obtenção do Título Mestre em Ciências da Saúde pelo Programa de Pós-Graduação em Ciências da Saúde da Universidade de Brasília
\end{abstract}

Orientadora: Profa. Dra. Janeth de Oliveira S. Naves Co-Orientadora: Profa. Dra. Erica Negrini Lia

BRASÍLIA 


\title{
EFEITO PREEMPTIVO DO IBUPROFENO NA DOR PÓS- OPERATÓRIA ASSOCIADA À EXODONTIA DE TERCEIRO MOLAR: ENSAIO CLÍNICO RANDOMIZADO DUPLO-CEGO CRUZADO COMPARANDO DOSES MÚLTIPLAS VERSUS DOSE ÚNICA
}

\begin{abstract}
Dissertação apresentada como requisito parcial para obtenção do Título Mestre em Ciências da Saúde pelo Programa de Pós-Graduação em Ciências da Saúde da Universidade de Brasília
\end{abstract}

Aprovado em: 02/03/2015

BANCA EXAMINADORA

Profa. Dra. Janeth de Oliveira Silva Naves - Presidente Universidade de Brasília

Profa. Dra. Ana Cristina Bezerra Universidade de Brasília

Profa. Dra. Dayde Lane Mendonça da Silva Universidade de Brasília

Prof. Dr. Sérgio Bruzadelli Macedo - Suplente Universidade de Brasília 
Dedico este trabalho a minha amada família, aos meus filhos, meu maior tesouro, Luís Felipe e Pedro Henrique que me iluminam e inspiram sempre, a minha filha Raquel que mesmo dentro de mim participou totalmente das etapas finais. Ao meu marido que sempre me incentivou e apoiou. Aos meus pais, Jerffson e Dirce, meu alicerce, e meus irmãos que sempre me incentivaram e torceram pela minha felicidade e sucesso, obrigada por tudo! Amo vocês. 


\section{AGRADECIMENTOS}

Agradeço a Deus pelo dom da vida, pela oportunidade de poder desfrutar momentos maravilhosos comas pessoas que amo e conhecer e conviver com pessoas especiais no meu caminho.

Á minha amada mãe Dirce e minha querida sogra Bernadete pelas inúmeras vezes que me ajudou com meus filhos para que eu pudesse estudar e me dedicar ao mestrado.

À Professora Janeth de Oliveira S. Naves, por ter me recebido como orientanda e pela confiança em mim depositada.

À Professora Érica Negrini Lia, co-orientadora e um exemplo para mim como professora. Obrigada por ter aceitado me co-orientar neste trabalho.

Ao Prof. Dr. Sérgio Bruzadelli Macedo, por ter me recebido tão atenciosamente, pelas cirurgias realizadas e pelo empenho e colaboração para que esta pesquisa se realizasse.

Ao Professor Eduardo Barbosa Coelho, pela valiosa contribuição na análise estatística ajuda fundamental na reta final. Obrigada.

Ao cirurgião dentista Mateus Veppo dos Santos, residente em cirurgia bucomaxilofacial, sempre pronto a ajudar, obrigada pela realização das cirurgias.

Ao Prof. André Luiz Vieira Cortez que me favoreceu a realização deste trabalho.

Aos funcionários da Universidade de Brasília que sempre colaboraram para a realização desta pesquisa.

Sem o apoio de vocês, este trabalho não teria sido possível! 
"Uma paixão forte por qualquer objeto assegurará o sucesso, porque o desejo pelo objetivo mostrará os meios." (William Hazlitt) 


\section{RESUMO}

A dor pós-operatória é a causa mais comum de hiperalgesia em humanos e pode ter sua severidade e duração reduzidas através da analgesia preemptiva, tratamento antinociceptivo que previne o processamento alterado de impulsos nervosos responsáveis pela sensibilização central. A literatura sugere que o uso préoperatório de anti-inflamatórios pode diminuir a intensidade e a duração da dor pósoperatória. Dezenove participantes submetidos à extração de terceiros molares inclusos inferiores bilaterais participaram de ensaio clínico randomizado, duplo-cego, cruzado, no qual foi comparado o uso de dose única (DU, 600mg de ibuprofeno, VO, 1 hora antes da cirurgia) versus doses múltiplas (DM, 600mg de ibuprofeno 25, 19, 13, 7 e 1 hora antes da cirurgia). O desfecho principal foi a redução da dor pósoperatória, medida pelo uso da escala visual analógica (EVA) aplicada imediatamente e 3, 8 e 24 horas após a cirurgia. Como desfechos secundários utilizaram-se a medida da redução do grau de abertura bucal $24 \mathrm{~h}$ pós-cirurgia, o consumo de analgésico socorro (Codeína $30 \mathrm{mg}$, VO) nas $24 \mathrm{~h}$ pós-cirurgia e a aplicação dos filamentos Semmes-Weinstein ( $\Delta$ log força g). O grupo DM apresentou menor intensidade de dor (EVA) quando comparado com o controle nos tempos $3 \mathrm{~h}$ e 8h após a cirurgia ( $22 \pm 21$ vs. $37 \pm 29 ; 27 \pm 22$ vs. $37 \pm 34$; DM VS DU, 3h e 8h respectivamente, $P=0,03)$. Não houve diferença com relação aos desfechos secundários. Em conclusão, o uso de doses múltiplas foi superior ao uso de dose única de ibuprofeno na analgesia preemptiva associada à cirurgia de remoção de terceiros molares inferiores inclusos.

Palavras-chave: dor pós-operatória; medição da dor; ibuprofeno; terceiro molar; hiperalgesia. 


\begin{abstract}
Postoperative pain management still represents a challenge for some clinicians, and its severity and duration can be reduced by preemptive analgesia, defined as an antinociceptive treatment that prevents the establishment of altered central processing of afferent input, responsible by amplification of postoperative pain. Nineteen participants underwent extraction of bilateral lower impacted third molars were enrolled in a randomized clinical, double-blind, crossover clinical trial, which was compared using single dose (SD, 600mg of ibuprofen, oral, 1 hour before surgery) versus multiple doses (MD, $600 \mathrm{mg}$ ibuprofen 25, 19, 13, 7 and 1 hour before surgery). The primary outcome was reduction of postoperative pain, measured by using a visual analogue scale (VAS) immediately and 3, 8 and 24 hours after surgery. Secondary outcomes were reduction of degree of mouth opening, consumption of rescue analgesics (codeine $30 \mathrm{mg}$, orally) and measurement of the pain threshold by Semmes-Weinstein filaments application ( $\Delta \log$ force $\mathrm{g}$ ), 24 hours after the surgery. The MD group showed less pain (VAS) compared with the control at 3 and 8 hours after surgery (22 \pm 21 vs. $37 \pm 29,27 \pm 22$ vs. $37 \pm 34$; MD vs. SD, $3 h$ and $8 \mathrm{~h}$ respectively, $\mathrm{P}=0.03)$. There was no difference with respect to the secondary outcomes. In conclusion, the use of multiple doses is superior to single dose of ibuprofen as preemptive analgesia strategy associated with surgical removal of impacted lower third molar.
\end{abstract}

Key words: Pain Postoperative; Pain Measurement; Ibuprofen; Molar, Third; Hyperalgesia. 


\section{LISTA DE FIGURAS}

Figura 1 Cartela contendo código alfanumérico e identificação da sequência de cápsulas a serem ingeridas

Figura 2 Escala Analógica Visual

Figura 3 Kit completo de filamentos Semmes-Weinstein

Figura 4 Filamento Semmes-Weinstein

Figura 5 Mensuração do grau de abertura bucal

Figura 6 Fluxograma do estudo

Figura 7 Desfecho principal: Redução da dor no período pós-operatório medida pela EVA comparando dose única (DU) e doses múltiplas (DM) de ibuprofeno. Valores apresentados como média $\pm \mathrm{DP}{ }^{*} \mathrm{p}<0.05$

Figura 8 Diferença entre as medidas algesimétricas realizadas com filamentos Semmes- Weinstein nos ponto P1 e P2 nos grupos DU versus DM. Valores expressos como log força em gramas (mediana e intervalo 25 - 75\%).

Figura 9 Comparação entre a redução da abertura bucal $(\mathrm{mm}) 24 \mathrm{~h}$ após a cirurgia

Figura 10 Proporção do uso de medicamentos de socorro entre os grupos DU e DM. $(15,8$ vs. $5,3 \%, P=0,60)$ 


\section{LISTA DE TABELAS}

Tabela 1 - Características cirúrgicas nos grupos DU e DM 


\section{LISTA DE ABREVIATURAS E SIGLAS}

AINEs - anti-inflamatórios não-esteróides

ANVISA - Agência Nacional de Vigilância Sanitária

CGRP - peptídeo relacionado ao gene da calcitonina

CONSORT - Consolidated Standards of Report Trials

COX-1- ciclooxigenase 1

COX-2- ciclooxigenase 2

DM - Dose múltipla

DU - Dose única

EPM - erro padrão da média

EVA - Escala visual analógica

EVN - Escala Visual Numérica

HUB - Hospital Universitário de Brasília

IASP - International Association for the Study of Pain

OMS - Organização Mundial de saúde

REBEC - Registro Brasileiro de Ensaios Clínicos

RENAME - Relação Nacional de Medicamentos Essenciais

VO - Via oral 


\section{SUMÁRIO}

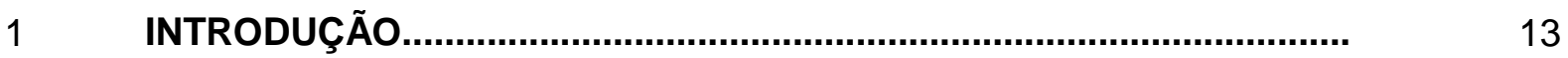

2 REVISÃO DA LITERATURA.................................................................





2.2.1 Mecanismos periféricos...................................................................... 14

2.2.2 Mecanismo central...................................................................................

2.3 MÉTODOS FARMACOLÓGICOS DE CONTROLE DA DOR



2.3.1 Analgesia Preemptiva........................................................................

2.4 EXODONTIAS DE TERCEIROS MOLARES RETIDOS............................ 20

2.5 MENSURAÇÃO DA DOR EM HUMANOS.............................................. 21

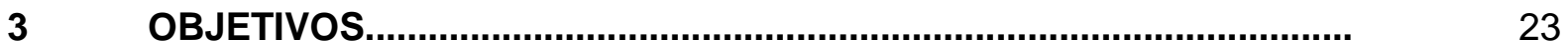



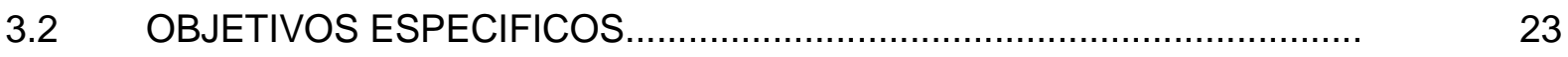

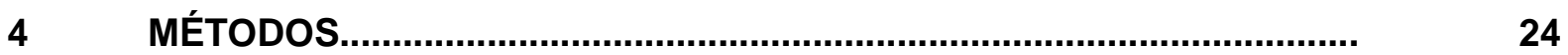

4.1 TIPO DE ESTUDO .................................................................... 24



4.3 DESFECHOS .............................................................................

4.4 CÁLCULO AMOSTRAL E CASUÍSTICA.............................................. 25

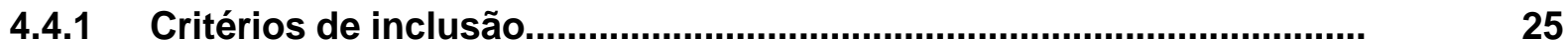

4.4.2 Critérios de exclusão................................................................................

4.5 PROTOCOLO DE PESQUISA............................................................

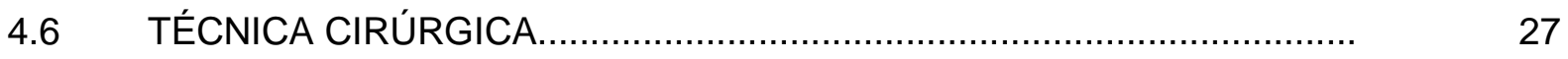

4.7 AVALIAÇÃO ALGESIMÉTRICA ....................................................... 28

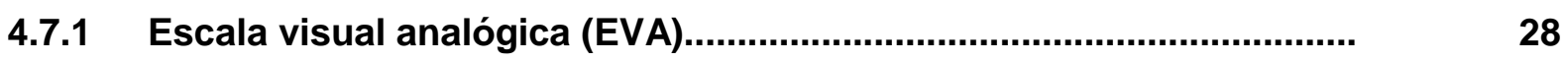

4.7.2 Filamentos Semmes-Weinstein............................................................ 28

4.7.3 Grau de abertura bucal..........................................................................

4.7.4 Consumo de analgésico socorro............................................................... 30

4.7.5 Eventos adversos dos medicamentos utilizados............................... 30

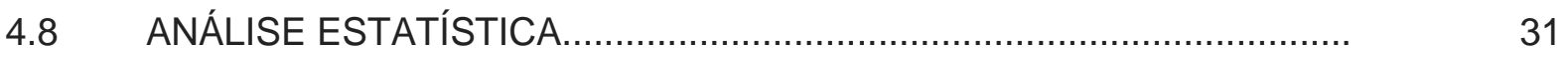

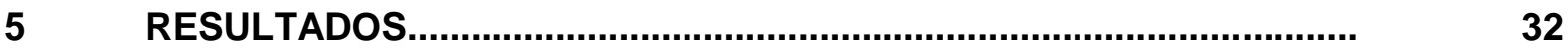

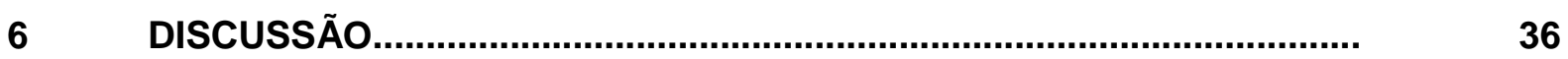

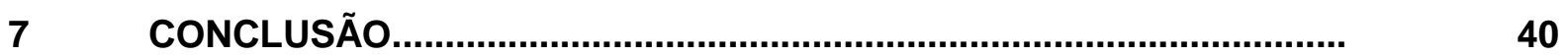

REFERENCIAS...............................................................................

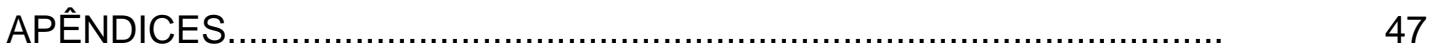


ANEXO 


\section{INTRODUÇÃO}

A dor pós-operatória ainda representa um desafio para muitos clínicos, e pode ter sua severidade e duração reduzidas por meio do emprego da analgesia preemptiva, definida como tratamento antinociceptivo que previne o estabelecimento do processamento alterado de impulsos nervosos aferentes, responsáveis pela amplificação da dor pós-operatória $(1,2)$.

Frente ao trauma tecidual, mediadores inflamatórios como as prostaglandinas são formados a partir da ação da ciclooxigenase 2 (COX-2) sobre o ácido araquidônico presente em membranas celulares rompidas. As prostaglandinas sensibilizam os nociceptores periféricos, facilitando a geração local de estímulos, caracterizando a sensibilização periférica. Fenômenos semelhantes podem ocorrer também na medula espinhal e gânglio trigeminal, gerando a sensibilização central, definida clinicamente pela projeção da área hiperalgésica aos tecidos vizinhos à área injuriada $(3,4)$.

A literatura científica sugere que a administração de anti-inflamatórios não esteroidais antes da cirurgia tem a possibilidade de inibir precocemente a produção de prostaglandinas, prevenindo, portanto a sensibilização periférica e central (5).

Um dos modelos de dor pós-operatória mais estudados em Odontologia é a exodontia de terceiros molares inclusos, sendo comumente administrados antiinflamatórios não esteroidais no período pré-operatório para o controle da dor e do trismo. Entretanto, fatores como o tempo e a via de administração, a utilização de instrumentos algesimétricos distintos e avaliação realizada em momentos diferentes podem explicar resultados divergentes $(6,7,8,9,10,11)$.

$\mathrm{Na}$ literatura há apenas um estudo que utilizou doses múltiplas orais prévias de cetoprofeno à realização da extração de terceiros molares, porém sem evidenciar benefício das mesmas sobre a analgesia pós-operatória $(12,13)$.

O ibuprofeno é um anti-inflamatório largamente empregado para o tratamento e prevenção da dor odontogênica $(5,9,14)$ e não existem estudos com doses múltiplas prévias à cirurgia odontológica. Diante do exposto, o objetivo deste trabalho foi avaliar a eficácia da analgesia preemptiva de dose única versus doses múltiplas de ibuprofeno sobre a dor pós-operatória após exodontia de terceiros molares inferiores inclusos. 


\section{REVISÃO DA LITERATURA}

\subsection{DOR}

Segundo a International Association for the Study of Pain (IASP), a dor é definida como uma experiência sensorial e emocional desagradável associada a dano tecidual real ou potencial ou descrita em termos que sugerem tal dano (15). A dor é um fenômeno complexo multidimensional que envolve emoções, deve ser compreendida como modelo biopsicossocial e não apenas uma sensação. É um fenômeno subjetivo, pois cada pessoa percebe a dor de forma diferente, difícil de quantificar e qualificar; não podendo ser caracterizada objetivamente, pois não existem marcadores biológicos que permitam a medida. A mesma lesão pode causar dores diferentes em indivíduos diferentes ou no mesmo indivíduo em momentos diferentes (16).

A dor é um fenômeno muito frequente na Odontologia, principalmente em situações que envolvem manipulação tecidual. O trauma advindo do ato cirúrgico odontológico implica em alterações fisiológicas e emocionais que, se forem adequadamente controladas melhoram a qualidade de vida no período pósoperatório reduzindo a morbidade e proporcionando maior conforto ao paciente, permitindo desta forma rápida recuperação e retorno precoce às suas atividades de vida diária (4). Entre as condições que podem afetar a recuperação e a qualidade de vida do indivíduo, a dor merece atenção.

\subsection{FISIOPATOLOGIA DA DOR}

\subsubsection{Mecanismos periféricos}

A dor resulta de um conjunto de experiências sensitivas, cognitivas e emocionais, associadas a respostas autonômicas e comportamentais, relacionadas à estimulação nociceptiva produzida por uma lesão (fratura, incisão cirúrgica, queimadura etc). De modo geral, na evolução dos quadros álgicos agudos há aumento inicial da intensidade da dor seguida pela redução gradual, relacionada à resolução do processo inflamatório e cicatrização da área lesionada (4). 
O estímulo nocivo de natureza mecânica, térmica, biológica ou química provoca dano tecidual, o que resulta no acúmulo de substâncias algogênicas (histaminas, prostaglandinas, bradicinina, entre outras). Estas substâncias sensibilizam ou ativam diretamente as terminações nervosas livres, as fibras $A \delta$ e $C$, que respondem por meio da deflagração de impulso nervoso, além de produzir neuropeptídeos, como a substância $\mathrm{P}$ e o peptídeo relacionado ao gene da calcitonina (CGRP), que agridem os tecidos, liberando mais mediadores inflamatórios, desencadeando assim o processo de sensibilização periférica $(3,4,17)$.

Prostaglandinas e leucotrienos encontrados nos sítios de inflamação participam como mediadores de vários fenômenos, como a degranulação de mastócitos e a ativação direta de fibras nervosas, macrófagos e linfócitos. Há liberação de mediadores, como potássio, serotonina, substância $P$, histamina e cininas. Ocorrem alterações da permeabilidade vascular, fluxo sanguíneo regional com a consequente produção dos sinais clássicos inflamatórios, como a hiperemia, aumento da temperatura local, aumento de volume, dor e eventualmente perda de função $(3,17)$.

Estudos clínicos e experimentais têm demonstrado que estímulos nocivos provocam alterações no sistema nervoso central, modificando os mecanismos desencadeados pelos estímulos aferentes $(3,17)$. A estimulação persistente de nociceptores provoca dor espontânea, redução do limiar de sensibilidade e hiperalgesia. A presença de todos esses elementos sugere que a sensibilização periférica não é o único fenômeno responsável por todas essas mudanças e que há envolvimento do sistema nervoso central neste processo $(3,17)$.

\subsubsection{Mecanismos centrais}

A dor inflamatória leva a quadros de hiperalgesia (aumento da sensibilidade dolorosa) ou alodinia (dor em resposta a um estímulo não-nociceptivo) (15). Além da hiperalgesia primária, que ocorre no local do trauma, há projeção da área hiperalgésica aos tecidos vizinhos, o que caracteriza a hiperalgesia secundária. Neste processo estão envolvidos os metabólitos do ácido araquidônico, substância $\mathrm{P}$, glutamato, histamina, citocinas e componentes plasmáticos (como a bradicinina) que aumentam a sensibilização das fibras nervosas periféricas por contribuírem para a liberação de substâncias neuroativas $(5,17)$. Este quadro leva ao aumento da 
excitabilidade dos neurônios de segunda ordem, localizados no corno dorsal de medula espinhal e no gânglio trigeminal (no caso das estruturas cefálicas). Esse fenômeno é conhecido como sensibilização central, mecanismo fisiopatológico da hiperalgesia secundária $(1,5,17)$.

\subsection{MÉTODOS FARMACOLÓGICOS DE CONTROLE DA DOR INFLAMATÓRIA}

Medicamentos como anti-inflamatórios não esteroidais (AINEs), analgésicos opióides e corticosteróides são empregados com o objetivo de controlar os sinais e sintomas exacerbados da resposta inflamatória, como edema, dor e febre. Os corticosteróides são anti-inflamatórios esteroidais que inibem a síntese da enzima fosfolipase A2, bloqueando, portanto a transformação de fosfolipídeos presentes na membrana celular em ácido araquidônico, impedindo a síntese subsequente de mediadores inflamatórios (prostaglandinas, prostaciclinas, leucotrienos) $(18,19)$.

Como todo medicamento, os corticoides também podem levar a efeitos adversos, dentre os quais se destacam hiperglicemia, glicosúria, miopatia, osteoporose, osteonecrose, retardo de crescimento, úlcera péptica, problemas oculares, alterações do sistema nervoso central, alteração na distribuição de gordura corporal, susceptibilidade às infecções, supressão da função adrenal e dificuldade no reparo tecidual. Deve-se ressaltar, entretanto, que esses efeitos são dependentes da potência do corticoide, da dose e, especialmente, da duração do tratamento $(18,19)$.

Os anti-inflamatórios não-esteroidais (AINEs), tais como os derivados do ácido acetilsalicílico, o ibuprofeno, a indometacina, os diclofenacos, o piroxicam, o rofecoxibe dentre outros, inibem a enzima ciclooxigenase, responsável pela síntese de prostaglandinas. Em 1990, foi demonstrada a existência de duas isoformas distintas da COX, denominadas COX-1 e COX-2 (20). A COX-1 é uma enzima constitutiva, presente em vários tecidos, responsável pela geração de prostaglandinas envolvidas em funções homeostáticas como citoproteção e agregação plaquetária. Já a COX-2, mantém-se em níveis basais, elevando-se na presença do processo inflamatório $(21,22)$.

Os efeitos adversos dos AINEs mais importantes podem ocorrer com longo período de uso. Há pacientes que exigem maior atenção, como os hipertensos que fazem uso de inibidores da enzima conversora de angiotensina, betabloqueadores 
ou diuréticos, pois a ação desses medicamentos é mediada por prostaglandinas renais que são diminuídas pela atuação dos anti-inflamatórios após 7 a 8 dias de uso; pacientes que fazem uso regular de anticoagulantes, pelo aumento potencial no sangramento transe pós-operatório e pacientes que apresentam decréscimo da função renal (21).

O ibuprofeno é o anti-inflamatório mais relatado na literatura americana, é um fármaco proveniente do ácido propiônico cuja aplicação também é indicada no controle da dor em cirurgias bucais $(23,24)$. É administrado por via oral, em doses de 300 a 600mg, a cada 6 horas, preferencialmente após as refeições. Tem início de ação 30 minutos após administração, leva 1 a 2 horas para ser absorvido por via oral e meia-vida de 2 horas (22). O ibuprofeno é amplamente usado em diversos países para o alívio dos sintomas de dor, febre e inflamação $(5,25)$. Na prática clínica odontológica é usualmente utilizado no período pós-operatório.

De acordo com estudos epidemiológicos, o ibuprofeno apresenta baixo risco gástrico, hepático e de lesão renal em relação a outros AINEs com a mesma eficácia analgésica (14). No Brasil, o ibuprofeno está integrado na Relação Nacional de Medicamentos Essenciais (RENAME) (26), lista elaborada por especialistas observando critérios de eficácia e segurança, entre outros, com base no paradigma da saúde baseada em evidências. E disponibilizado no componenete básico da atenção à saúde.

\subsubsection{Analgesia Preemptiva}

A analgesia preemptiva baseia-se na hipótese de que o bloqueio dos fatores que levam à sensibilização central apresenta efeito positivo sobre a diminuição da dor pós-operatória (5). Como ferramenta de grande destaque, a analgesia preemptiva consiste na instituição de um regime medicamentoso prévio ao estímulo nociceptivo, com o objetivo de prevenir a hiperalgesia e a subsequente amplificação da dor (27), ou seja, antes da incisão $(2,28)$.

Crile (1913) introduziu o conceito de analgesia preemptiva com base em observações clínicas da utilização de bloqueios regionais associados à anestesia geral para impedir o estímulo nociceptivo no período transoperatório. Notou a diminuição na frequência cardíaca, redução do sinal de febre e a mortalidade pósoperatória (29). 
Cliff e colaboradores (30) realizaram metanálise a partir de 66 ensaios clínicos randomizados sobre analgesia preemptiva na dor pós-operatória, totalizando 3.261 pacientes. Compararam o tratamento com placebo ou nenhum tratamento préoperatório; tratamento pré-operatório com uma combinação de tratamento préoperatório mais pós-operatório, e a comparação de pré-operatório diferente do regime de tratamento pós-operatório. Para avaliação da intensidade da dor utilizaram a escala visual analógica (EVA) pontuada durante as primeiras 24-48 horas pós-operatórias, a necessidade de analgésicos no período pós-operatório suplementar e o tempo para a primeira tomada do analgésico de socorro. A conclusão demonstrou a possível eficácia de analgesia preemptiva para melhorar a dor pós-operatória. O efeito preemptivo da analgesia resultou em melhorias consistentes em todas as três variáveis de resultado (30).

Na prática clínica também são utilizados outros conceitos como analgesia preventiva e multimodal $(17,28)$. Garcia e colaboradores (17) e Rosero (28) definem a analgesia preventiva, como aquela que é realizada em qualquer momento (antes, durante ou após a cirurgia) para evitar o aparecimento ou diminuir da intensidade da dor. A analgesia multimodal consiste na administração de dois ou mais fármacos que atuam por diferentes mecanismos para proporcionar analgesia. Estes fármacos podem ser administrados pela mesma via ou por vias diferentes e foi desenvolvido com base no conhecimento de que a dor pós-operatória é um fenômeno complexo e multifatorial, e que a utilização de substâncias que atuam por diferentes mecanismos de ação apresentam efeito analgésico sinérgico (28).

A maioria dos ensaios clínicos randomizados comparando o efeito da administração pré-operatória de vários medicamentos em relação ao tratamento pós-operatório, incluindo medicamentos anti-inflamatórios não-esteróides (AINEs), não demonstrou diferenças significativas das mensurações algesimétricas. As razões para a falta de benefício desses resultados podem incluir a utilização de um único medicamento (em contraste com uma abordagem multimodal) ou a utilização de uma única dose de analgésico, que pode ter resultado de duração inadequada da analgesia (28).

O uso pré-operatório de AINEs parece ser um método válido para aumentar o conforto pós-operatório do paciente, reduzindo o uso de medicamentos analgésicos, diminuindo a intensidade da dor e a intensidade do edema $(9,10,27,31,32)$. Existem 
evidências sobre sua efetividade, porém ainda falta a racionalização e divulgação do seu uso (27).

Os resultados de estudos clínicos sobre a analgesia preemptiva não são homogêneos, pois dependem do tempo de administração prévio ao procedimento cirúrgico, tipo de droga utilizada, dosagem, via de administração, tempo de avaliação da dor pós-operatória e diferentes métodos de avaliação algesimétrica $(9,10)$. Alguns estudos não conseguiram constatar o efeito preemptivo da utilização prévia de antiinflamatórios não esteroidais à cirurgia para extração de terceiros molares inclusos $(11,12,13)$. Outros estudos evidenciaram o efeito preemptivo de anti-inflamatórios não esteroidais para o mesmo tipo de cirurgia e verificaram maior eficácia analgésica com a utilização do celecoxibe em comparação com um anti-inflamatório convencional (33).

Kissin (2000) afirma que a analgesia pré, trans e pós-operatória é necessária para prevenir a sensibilização central, pois em estudos comparativos de grupos nos quais o analgésico é administrado apenas no período pré-incisional e pós-incisional, não é possível detectar efeito preemptivo devido à complexidade da sensibilização central e às dificuldades técnicas para os estudos clínicos (1).

Liporaci Junior (2012) comparou a eficácia analgésica da administração pósoperatória de cetoprofeno por via oral, com a administração pré-operatória, iniciada dois dias antes da cirurgia de extração de terceiros molares. A despeito do pequeno número de pacientes inseridos na pesquisa, os resultados observados nesse trabalho demonstraram não haver diferença significativa entre os esquemas pré e pós-operatório do cetoprofeno frente à cirurgia de remoção de terceiros molares inclusos (12).

A prescrição de analgésicos em horário fixo evita grandes oscilações do nível plasmático do fármaco e previne picos de dor(4). Para o sucesso do tratamento é necessário garantir que o medicamento escolhido atinja o alvo em concentrações adequadas. Para tal, é necessário escolher doses, vias de administração e intervalos entre doses que garantam que as concentrações terapêuticas atinjam o sítio-alvo e aí se mantenham (34).

Outros fatores como idade, gênero, história médica, presença de pericoronarite, má higiene oral, técnica cirúrgica, antissepsia, experiência do cirurgião, uso de antibióticos no perioperatório ou de medicamentos intra-alveolares, 
qualidade e quantidade do anestésico e técnica anestésica também podem influenciar significativamente a resposta dolorosa desse tipo de intervenção $(35,36)$.

Vários estudos foram realizados demostrando a eficácia dos AINEs no controle da dor pós-operatória, inclusive quando administrados no pré-operatório. Considera-se que a melhor e mais fácil maneira de tratar uma complicação, como a dor, é prevenir que ela aconteça, no entanto, uma vez identificada, esta deverá ser efetivamente tratada $(9,10,27,31,32,37)$.

\subsection{EXODONTIAS DE TERCEIROS MOLARES RETIDOS}

A cirurgia de remoção de terceiros molares é o procedimento mais usado para avaliar dor pós-operatória, sendo reconhecido internacionalmente, pois resulta em dor moderada a intensa e permite um estudo prospectivo, aleatório, duplo-cego, com pareação de amostras na qual o paciente poderá atuar como seu próprio controle, sofrendo intervenção em momentos diferentes (13,38). Um ponto importante que deve ser observado é a obediência aos princípios de técnica cirúrgica, pois este minimiza as reações adversas do trauma operatório favorecendo o controle da dor (36).

Para facilitar o planejamento e a comunicação entre os cirurgiões-dentistas, surgiram algumas classificações dos terceiros molares que permitem a antecipação de possíveis transtornos e a previsão de algumas modificações durante 0 ato operatório; tais classificações são feitas a partir da análise radiográfica $(39,40)$.

Winter (1926) classificou os terceiros molares inferiores baseando-se na angulação do longo eixo do dente incluso em relação ao longo eixo do segundo molar, desta forma, podem encontrar-se na posição vertical, mesio-angular, distoangular, horizontal, invertida e ainda em línguo-versão ou vestíbulo-versão $(40,41)$.

A Classificação de Pell \& Gregory (1973) relaciona a superfície oclusal dos terceiros molares inferiores à posição do segundo molar adjacente (posição $A, B, C$ ) e o diâmetro mesio-distal do terceiro molar em relação à borda anterior do ramo da mandíbula (Classe I, II e III) (40). Na posição A, o dente encontra-se acima do plano oclusal ou na mesma linha do segundo molar inferior. Na posição $\mathrm{B}$, o dente encontra-se abaixo do plano oclusal e acima da linha cervical do segundo molar inferior. Na posição $\mathrm{C}$, o dente encontra-se abaixo da linha cervical do segundo molar inferior. Na classe I há espaço suficiente entre o ramo ascendente da 
mandíbula e o segundo molar para acomodação do $3^{\circ}$ molar (incluso). Na classe II, o espaço entre o ramo ascendente e a distal do segundo molar é menor que o diâmetro mesio-distal do $3^{\circ}$ molar, há pouco espaço para a acomodação do $3^{\circ}$ incluso. Na classe III, o $3^{\circ}$ molar está quase ou totalmente incluso no ramo ascendente da mandíbula. Há pouco ou nenhum espaço para a acomodação do dente (42).

\subsection{MENSURAÇÃO DA DOR EM HUMANOS}

A intensidade da dor é percebida de forma distinta entre diferentes indivíduos, e pode ser modificada pela personalidade e estado psicológico. O estado de ansiedade potencializa a dor, aumenta sua percepção e diminui sua tolerância, gerando tensão muscular, o que produz o círculo "dor-tensão-dor"; isso conduz ao raciocínio de que, de forma alheia a uma causa orgânica, é certo que vários elementos incidem sobre a dor $(38,40)$.

Os instrumentos para mensurar a dor em humanos podem ser unidimensionais ou multidimensionais. Escalas unidimensionais avaliam somente uma das dimensões da experiência dolorosa, e dentre as mais usadas por clínicos e pesquisadores, destacam-se a Escala Visual Numérica (EVN), graduada de zero a dez, nas quais zero significa ausência de dor e dez, a pior dor imaginável; e a Escala Visual Analógica (EVA), que consiste de uma linha reta, não numerada, indicando-se em uma extremidade a marcação de "ausência de dor", e na outra, "pior dor imaginável" $(43,44)$. O questionário da dor de McGill e a escala de Minesota são exemplos de escalas multidimensionais para avaliação da intensidade e duração da dor.

Silva e Deliberato concluíram em sua revisão da literatura que, apesar de as escalas de dor não conseguirem mensurar com total precisão esse sintoma, devido à sua subjetividade e aos fatores que o influenciam, é a alternativa mais segura para analisá-lo, uma vez que estão validadas e demonstram ser confiáveis (38).

Há métodos para avaliação de estados de hipoalgesia ou hiperalgesia em pele e mucosas, dentre ele, os monofilamentos de Semmes-Weinstein, constituídos por fios de teflon de comprimentos e calibres diferentes. São utilizados para determinar parâmetros de origem tátil, mecânica e para quantificar objetivamente processos nociceptivos (45). Cada um deles está relacionado com uma força 
específica para curvá-lo, que varia de 0,008g a 300,0g no conjunto de vinte monofilamentos. Quanto maior o diâmetro do fio, maior será a força necessária para curvá-lo. A aplicação de estímulos com forças progressivas permite avaliar e quantificar o limiar de percepção do tato e pressão, estabelecendo correspondência com os níveis funcionais $(46,47,48)$. A deformação de suas hastes é transformada em parâmetros (logaritmos) de força com unidade em gramas, fazendo com que a avaliação da intensidade da dor deixe de ser subjetivo e passe a ter um caráter mais direto (45).

O grau de abertura bucal ou distancia interincisal tem sido utilizada como uma medida validada para mensuração do trismo mandibular em vários estudos $(40,49)$. A incapacidade de abrir a boca pode ser causada pelo hematoma ou inflamação dos tecidos moles no pós-operatório, ou ainda, por um espasmo muscular reflexo através de um processo inflamatório, após remoção de terceiros molares inferiores (40).

A dor inflamatória é uma importante causa de morbidade pós-operatória; entretanto, ainda existem lacunas relacionadas aos estudos sobre analgesia preemptiva em humanos e sua aplicabilidade clínica. Não há consenso sobre o protocolo medicamentoso pré-operatório que apresente melhor eficácia no controle da dor após a remoção de terceiros molares inferiores retidos. Não há estudos comparativos entre dose única e doses múltiplas orais pré-operatórias de ibuprofeno sobre o controle da dor pós-operatória. 


\section{OBJETIVOS}

\subsection{OBJETIVO GERAL}

Avaliar a eficácia da analgesia pré-operatória em dose única versus doses múltiplas de ibuprofeno no controle da dor pós-operatória após extração de terceiros molares inclusos.

\subsection{OBJETIVOS ESPECÍFICOS}

- Verificar a ocorrência de eventos adversos decorrentes do uso da analgesia pré-operatória em dose única versus doses múltiplas de ibuprofeno.

- Avaliar a influência do uso de dose única versus múltiplas doses de ibuprofeno sobre o grau de abertura da bucal. 


\section{MÉTODOS}

\subsection{TIPO DE ESTUDO}

Ensaio clínico randomizado, cruzado, duplo-cego, controlado por placebo.

\subsection{ASPECTOS ÉTICOS}

O projeto foi aprovado pelo Comitê de Ética em Pesquisa de Seres Humanos da Faculdade de Ciências da Saúde da Universidade de Brasília (CAAE 14533413.3.0000.0030) (Anexo A) e registrado na REBEC (Registro Brasileiro de Ensaios Clínicos), UTN U1111-1156-4165.

Os participantes que preencheram os critérios de inclusão foram convidados a conhecer a pesquisa, sendo dado a eles o direito de se recusarem a participar sem qualquer prejuízo para o seu atendimento. Foram esclarecidos os objetivos e a finalidade da pesquisa, assim como Ihes foram assegurados a confidencialidade, a privacidade, a proteção de imagem e a não-estigmatização. Todos os participantes receberam explicação acerca da pesquisa e manifestaram a concordância em participar da mesma, por meio da assinatura do Termo de Consentimento Livre e Esclarecido (Apêndice A). Todos receberam tratamento analgésico, pré e pósoperatório.

\subsection{DESFECHOS}

Considerou-se como desfecho primário a redução da dor pós-operatória verificada por meio da Escala Visual Analógica. A escala consiste em uma linha com 100 milímetros de comprimento, desenhada em uma folha de papel com a indicação "sem dor" e "pior dor possível" em suas extremidades. De acordo com a escala de dor da OMS, valores abaixo de 3 indicam dor de intensidade fraca e acima de 3 , dor de intensidade moderada $(44,50)$.

Como desfechos secundários foram estudados a redução do grau de abertura bucal (basal - 24h), a mensuração do limiar de dor pelo uso de filamentos SemmesWeinstein e a necessidade de uso de medicamentos de socorro. 
O ibuprofeno foi o anti-inflamatório escolhido por ser o mais citado na literatura americana tendo sua aplicação indicada no pré-operatório de cirurgias bucais $(2,24)$ e para o alívio dos sintomas de dor, febre e inflamação $(14,31)$.

Este fármaco tem início de ação 30 minutos após administração e meia-vida de 2 horas (22). O esquema posológico comparativo escolhido em dose única se deve por ser o mais usual na literatura $(11,21,31)$. O esquema dose múltiplas foi escolhido porque segundo alguns autores a prescrição de analgésicos em horário fixo evita grandes flutuações no nível plasmático do fármaco e previne picos de dor (4). Para o sucesso do tratamento é necessário garantir que o medicamento escolhido atinja o local afetado em concentrações adequadas, e escolher doses, vias de administração e intervalos entre doses que garantam que as concentrações terapêuticas atinjam o sítio-alvo e aí se mantenham (34).

\subsection{CÁLCULO AMOSTRAL E CASUÍSTICA}

O cálculo amostral foi realizado considerando uma diferença entre os grupos de ao menos $15 \mathrm{~mm}$ na EVA em favor do uso preemptivo de múltiplas doses de ibuprofeno, com valor de alfa igual a 0,05 e poder do teste de $80 \%$. Com estas considerações, o tamanho amostral mínimo de 17 participantes foi obtido. Participaram deste estudo 20 pacientes do Centro de Cirurgia Buco-Maxilo da Clínica Odontológica do Hospital Universitário de Brasília (HUB).

\subsubsection{Critérios de inclusão}

Pacientes de ambos os sexos, com idade superior a 18 anos, portadores de boa saúde geral e com indicação de exodontia bilateral de terceiros molares inclusos, inferiores, em posição similar de ambos os lados segundo a classificação radiográfica de Winter (1926) e Pell\& Gregori (1937).

\subsubsection{Critérios de exclusão}

Pacientes que não desejaram participar da pesquisa, sob uso de qualquer medicamento que atuava no mecanismo da dor nos 15 dias antecedentes ao início do estudo, gestantes, lactantes e com história de alergias ou efeitos adversos aos 
medicamentos utilizados no estudo, além da presença de pericoronarite relacionada ao terceiro molar inferior.

Pacientes que reportaram história de doença péptica pregressa ou sangramento digestivo prévio, insuficiência renal conhecida, doença hepática avançada e coagulopatias foram excluídos do estudo.

\subsection{PROTOCOLO DE PESQUISA}

Todos os participantes do estudo foram submetidos à remoção bilateral de terceiros molares inclusos intraósseos, em duas etapas, com intervalo mínimo de 30 dias entre cada uma, no período compreendido entre março e maio de 2014.

Na primeira cirurgia cada participante foi alocado aleatoriamente em um grupo e na segunda cirurgia entrava em outro grupo, atuando desta forma como seu próprio controle. O Grupo DU recebeu por via oral dose única de $600 \mathrm{mg}$ de ibuprofeno mais 4 doses de placebo e o Grupo DM recebia doses múltiplas de $600 \mathrm{mg}$ de ibuprofeno nos tempos 25, 19, 13, 7 e 1 hora antes da cirurgia.

O ibuprofeno é comercializado em doses de 200, 400 e 600 mg (dose plena), neste trabalho foi utilizado $600 \mathrm{mg}$ devido ao trauma intenso gerado pela remoção do terceiro molar incluso.

Por meio do programa True Randon Number Service, desenvolvido pela Escola de Ciência da Computação e Estatística de Dublin (Irlanda) e disponibilizado pelosite www.random.org gerou-se uma tabela de aleatorização com 40 códigos alfanuméricos. Cada código correspondeu a uma cartela com dose única de ibuprofeno e placebo ou doses múltiplas de ibuprofeno.

As cartelas foram preparadas e identificadas com os códigos por um farmacêutico e cada paciente recebeu o envelope codificado com a sequência de cápsulas a serem ingeridas. A pesquisadora principal, os cirurgiões e os participantes não tiveram acesso aos códigos de aleatorização durante a realização das cirurgias e tabulação dos dados.

O ibuprofeno e o placebo foram preparados por uma farmácia de manipulação e acondicionados em cápsulas idênticas (Figura 1). 


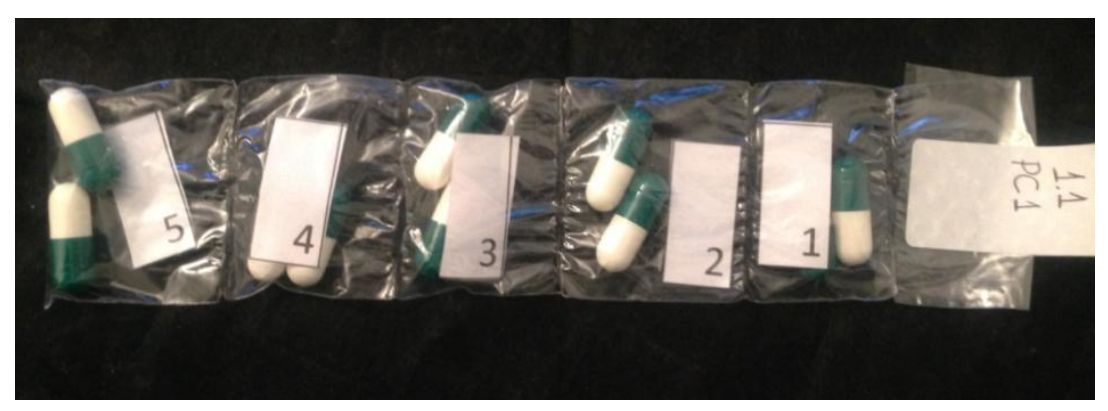

Figura 1 Cartela contendo código alfanumérico e identificação da sequência de cápsulas a serem ingeridas

\subsection{TÉCNICA CIRÚRGICA}

A remoção de terceiros molares inferiores inclusos é reconhecido internacionalmente como modelo de avaliação de dor aguda, é bastante utilizado em ensaios clínicos para o estudo da analgesia por induzir a dor de intensidade suficiente para que 95\% dos pacientes necessitem usar analgésicos (37).

Inicialmente realizou-se a antissepsia intraoral através do bochecho vigoroso, durante 1 minuto com solução de digluconato de clorexidina a 0,12\% e antissepsia extraoral com solução de povidine tópico.

A anestesia local foi realizada por meio do emprego do cloridrato de lidocaína associada à adrenalina 1:100.000 para o bloqueio dos nervos alveolar inferior, lingual e bucal. Utilizou-se seringa carpule com refluxo, agulha longa (Unoject, DFL, Brasil).

As cirurgias para remoção dos terceiros molares foram realizadas por 2 (dois) cirurgiões-dentistas, sendo um operador e um auxiliar, obedecendo a técnica descrita na literatura (40) e seguindo os procedimentos: Incisão mucoperiostal linear e relaxante vestibular com lâmina de bisturi número 15, descolamento mucoperiostal, osteotomia e odontossecção com broca tronco-cônica número 702 montada em alta rotação, sob irrigação abundante com soro fisiológico estéril $(\mathrm{NaCl}$ 0,9\%). A remoção do dente foi realizada com extratores tipo Seldin (número 2, 1R ou $1 \mathrm{~L})$, para curetagem foram utilizadas curetas e a remoção de espículas com limas para osso. Finalizou-se com a irrigação da loja cirúrgica, coaptação das bordas e sutura com fio de seda 3-0 (Shalon, Sertix, Brasil).

As cirurgias foram realizadas sempre pelo mesmo operador e todas as medidas foram realizadas pela pesquisadora principal. 
O tempo para realização das cirurgias foi anotado, sendo considerado seu início o momento da primeira incisão e como término o final da realização da sutura. O número de tubetes anestésicos, a frequência de osteotomia e odontossecção também foi anotada.

Todos os participantes receberam orientações pós-operatórias quanto aos cuidados locais e gerais (Apêndice B).

\subsection{AVALIAÇÃO ALGESIMÉTRICA}

\subsubsection{Escala analógica visual (EVA)}

A escala visual analógica (44) foi aplicada imediatamente e 3, 8 e 24 horas após o término da cirurgia. A escala consiste em uma linha com 100milímetros de comprimento, desenhada em uma folha de papel com a indicação "sem dor" e "pior dor possível" em suas extremidades (Figura 2).

O paciente foi orientado a realizar marcação sobre a linha, de acordo com a intensidade de dor percebida. O pesquisador realizou a medida da distância em milímetros entre a extremidade esquerda (sem dor) até a marca anotada pelo paciente, com o auxílio de uma régua.

Sem dor

Pior dor possível

Figura 2 Escala Analógica Visual

\subsubsection{Filamentos Semmes-Weinstein}

Foram aplicados pelo cirurgião os filamentos Semmes-Weinstein (Stoelting, USA) em dois pontos intrabucais (um ponto na região oclusal da loja cirúrgica suturada - P1 e outro ponto na região de fundo de vestíbulo da região do terceiro molar extraído - P2), imediatamente antes (medida basal) e 24 horas após a cirurgia. Foi anotado o filamento de menor calibre cuja aplicação em ângulo reto na superfície testada, seguido de sua dobra resultou em relato de dor pelo paciente. $O$ resultado foi expresso como o logaritmo da diferença entre as medidas 24 horas e basal. 
Os filamentos Semmes-Weinstein (Figuras3 e 4) constituem um grupo de 20 filamentos de teflon, de 38 milímetros de comprimento e diversos calibres. 0 princípio para utilização deste "kit" consiste em aplicar a extremidade do filamento em ângulo reto sobre a superfície a ser testada até que o mesmo se dobre. Cada um deles está relacionado com uma força específica para curvá-lo, que varia de 0,008g a 300,0g. Foram introduzidos em 1962 para avaliar a percepção tátil cutânea e testar estados de hipoalgesia ou hiperalgesia em pele e mucosas. Quanto maior o diâmetro do fio, maior será a força necessária para curvá-lo no momento em que é aplicado sobre a superfície a ser testada (29).

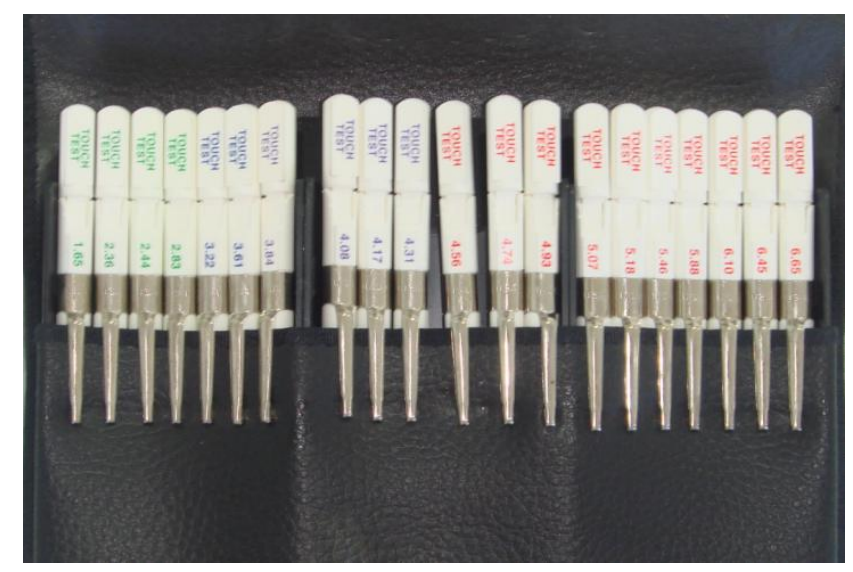

Figura 3 Kit completo de filamentos Semmes-Weinstein

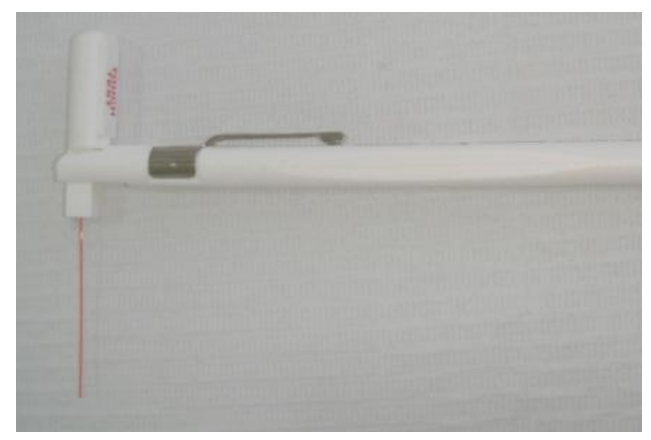

Figura 4 Filamento Semmes-Weinstein

\subsubsection{Grau de abertura bucal}

O grau de abertura bucal foi mensurado por meio da medida da distância interincisiva em milímetros (borda do incisivo central superior e inferior), durante a abertura máxima de boca alcançada, imediatamente antes e 24 horas após a 
cirurgia. O resultado foi reportado como a redução da abertura bucal, medida pela diferença entre o valor encontrado nas 24h após a cirurgia e o valor basal (Figura 5).

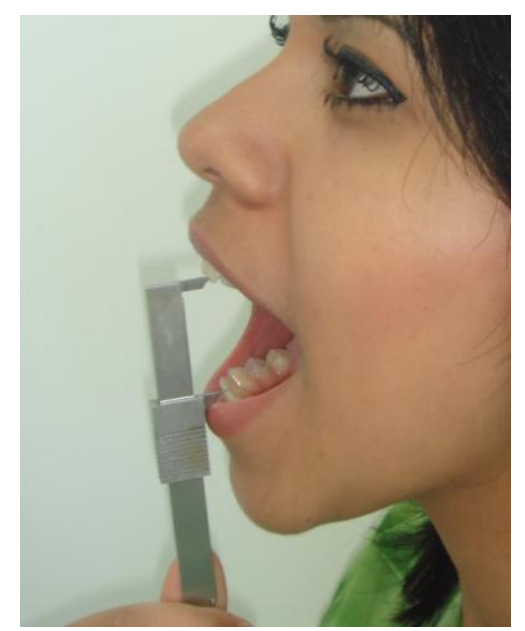

Figura 5 Mensuração do grau de abertura bucal

\subsubsection{Consumo de analgésico socorro}

Todos os participantes receberam 12 comprimidos de paracetamol $750 \mathrm{mg}$ com orientação para administrá-los por via oral a cada 6 horas durante três dias no período pós-operatório. Caso houvesse falha analgésica com o paracetamol, 24 horas após a cirurgia, o paciente recebia comprimidos de $30 \mathrm{mg}$ de codeína como analgésico socorro. Foi recomendado que a utilização de deste analgésico fosse cuidadosamente anotada.

O paracetamol foi escolhido por produzir analgesia por mecanismo semelhante aos salicilatos, inibindo a cicloxigenase no sistema nervoso central, diferente do ibuprofeno. Há evidências de que o paracetamol tenha fraca atividade anti-inflamatória em algumas condições não-reumatóides, por exemplo, em pacientes submetidos à cirurgia bucal (53).

\subsubsection{Eventos adversos dos medicamentos utilizados}

A definição de evento adverso seguiu a preconizada pela OMS e adotada pela ANVISA, ou seja, "qualquer ocorrência médica indesejável que ocorra com um paciente que tenha recebido um produto farmacêutico e que não necessariamente tenha relação causal estabelecida com este tratamento. Um evento adverso inclui 
qualquer sinal desfavorável e não intencional (achados laboratoriais anormais, por exemplo), sintomas, ou doença temporariamente associada com 0 uso do medicamento, relacionado ou não ao medicamento (51). Desta forma, foi recomendado que o paciente anotasse toda queixa clínica ou intercorrência que apresentasse nos 3 dias seguintes à cirurgia.

\subsection{ANÁLISE ESTATÍSTICA}

Os dados quantitativos com distribuição paramétrica foram reportados como média e desvio padrão ou erro padrão da média (EPM) ou como mediana e percentil 25 a 75\% para aqueles com distribuição não paramétrica. Dados qualitativos foram reportados como porcentagem e intervalo de variação mínima e máxima e foram comparados pelo teste exato de Fisher. Os resultados da EVA foram comparados por ANOVA two-way.

A redução do grau de abertura bucal e os resultados da utilização dos filamentos Semmes-Weinstein foram comparados pelo teste Mann-Whitney.

$\mathrm{O}$ índice de significância considerado foi para $\mathrm{P}<0,05$.

Para análise, utilizou-se o software estatístico Prism (versão 5.1,GraphPad, Inc., La Jolla, USA). 


\section{RESULTADOS}

No período de março a maio de 2014, foram selecionados sequencialmente vinte participantes que preencheram os critérios de inclusão e apenas um foi excluído por não ter preenchido adequadamente o formulário da EVA na primeira cirurgia. Entre os participantes, 7 eram do gênero masculino (37\%) e 12 eram do gênero feminino (63\%) com idade média de 22 anos ( \pm 4 ; $\min =18$; $\max =29)$. A Figura 6 mostra o fluxograma do recrutamento de participantes de acordo com as orientações do Consolidated Standards of Report Trials (CONSORT) para ensaios clínicos.

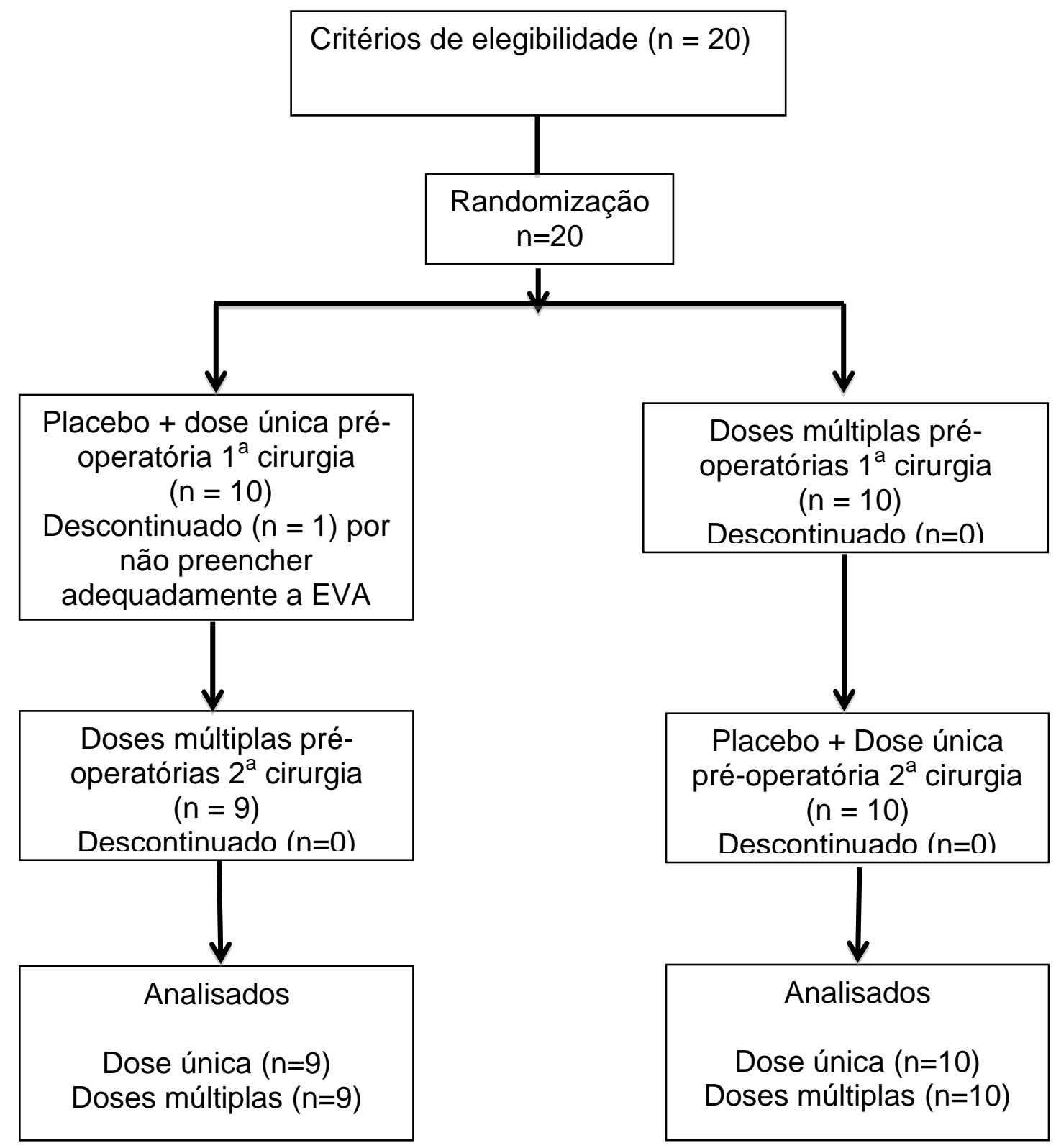

Figura 6 Fluxograma do estudo 
O tempo de execução de cada cirurgia foi semelhante e durou em média 22 minutos. Todos os procedimentos cirúrgicos ocorreram sem complicações. A Tabela 1 mostra as caraterísticas cirúrgicas. Não houve diferença significativa entre os grupos estudados (DU e DM) em relação os parâmetros cirúrgicos, como o tempo cirúrgico, número de tubetes de anestésicos utilizados e frequência de osteotomia e odontossecção. Dados expressos como média e desvio padrão para variáveis quantitativas paramétricas e como porcentagem para variáveis qualitativas.

Tabela 1 - Características cirúrgicas nos grupos DU e DM

\begin{tabular}{lccc}
\hline Variável & $\mathrm{DU}(19)$ & $\mathrm{DM}(19)$ & $\mathrm{P}$ Valor \\
\hline Tempo cirúrgico (min) & $21 \pm 10$ & $22 \pm 8$ & 0,81 \\
Tubete anestésico local (n) & $2,7 \pm 0,8$ & $2,5 \pm 0,6$ & 0,21 \\
Frequência osteotomia (\%) & 84 & 89 & 0,99 \\
Frequência odontossecção (\%) & 79 & 68 & 0,71 \\
\hline
\end{tabular}

Nas verificações três e oito horas após a cirurgia observou-se menores escores da EVA no grupo DM comparado ao grupo DU (22 \pm 21 vs. $37 \pm 29 ; 27 \pm 22$ vs. $37 \pm 34$; dose múltipla vs.dose única, $3 \mathrm{~h}$ e $8 \mathrm{~h}$ respectivamente, $P=0,03$ ). $A$ Figura 7 mostra a elevação significativa do escore da EVA em ambos os grupos, imediatamente após a cirurgia (tempo zero) $(\mathrm{P}<0.0001)$.

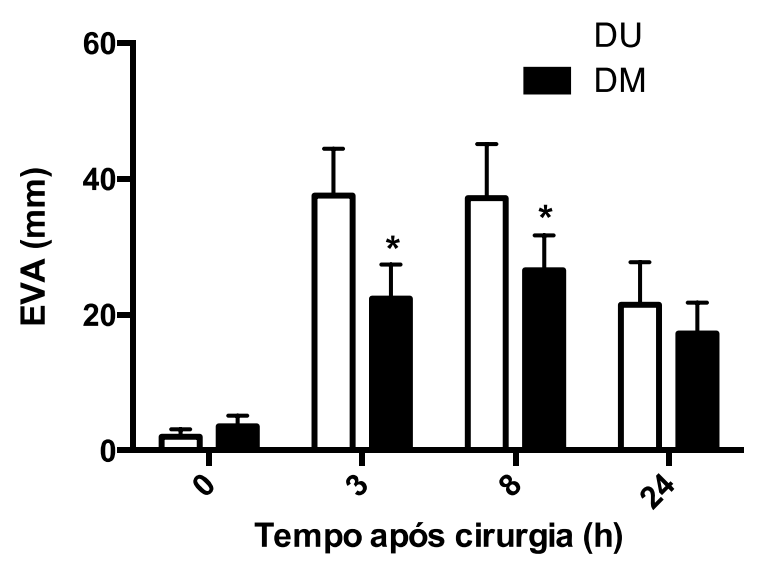

Figura 7 Desfecho principal: Redução da dor no período pós-operatório medida pela EVA comparando dose única (DU) e doses múltiplas (DM) de ibuprofeno. Valores apresentados como média $\pm D P{ }^{*} p<0.05$ 
A Figura 8 mostra os resultados obtidos a partir da aplicação dos filamentos Semmes-Weinstein. Não foram encontradas diferenças significativas entre os pontos $P 1$ e $P 2$, em ambos os grupos $(P 1=2,188(1,929-2,455)$ vs $2,079(1,903-2,380) p=$ 0,$5755 ; \mathrm{P} 2=2,188(1,903-2,380)$ vs $2,079(1,903-2,380) \mathrm{p}=0,7648$, DU vs DM respectivamente, $\Delta$ log força $\mathrm{g}$, mediana e intervalo 25 - 75\%). O uso dos filamentos não captou diferenças significativas na intensidade de dor antes e depois da cirurgia em ambos os grupos.



Figura 8 Diferença entre as medidas algesimétricas realizadas com filamentos SemmesWeinstein nos ponto P1 e P2 nos grupos DU versus DM. Valores expressos como log força em gramas (mediana e intervalo $25-75 \%$ ).

Não houve diferença significativa entre os grupos relacionada à redução da abertura bucal 24 horas após a cirurgia $(19(14-23) \mathrm{mm}$ vs $\mathrm{DM}=20(11-23) \mathrm{mm}$, DU vs DM, Valores de mediana e intervalo $25-75 \%, p=0,73$ ) (Figura 9).

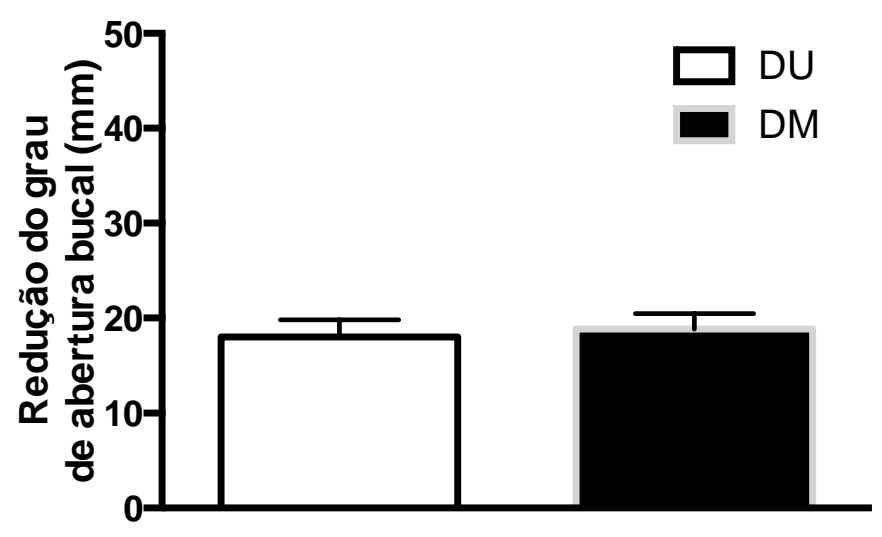

Figura 9 Comparação entre a redução da abertura bucal $(\mathrm{mm}) 24 \mathrm{~h}$ após a cirurgia.

O grupo DU apresentou maior tendência de utilização do analgésico socorro, embora não tenha sido observada diferença estatística significativa entre os grupos 
(15,8 vs. 5,3\%, $P=0,60$ ) (Figura 10). No grupo DU, três participantes utilizaram o analgésico socorro e no grupo DM apenas um utilizou.

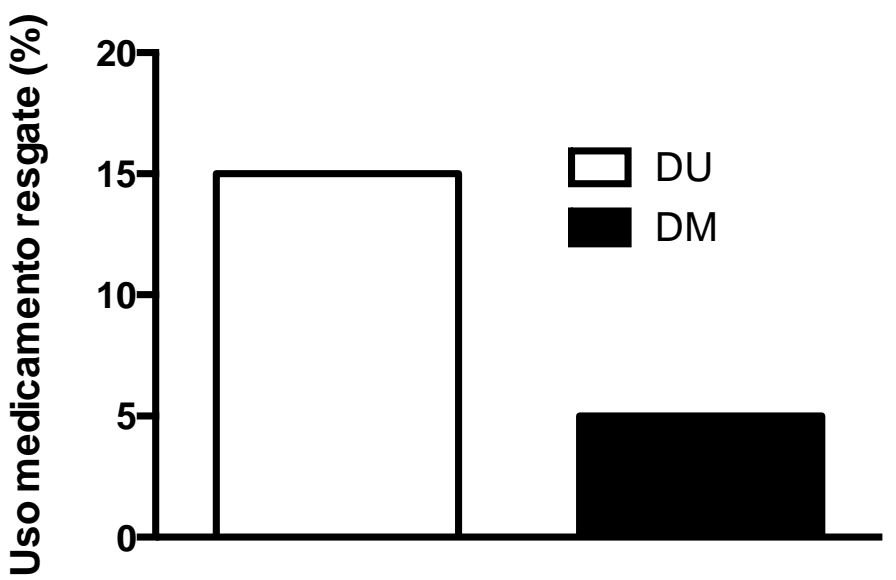

Figura 10 Proporção do uso de medicamentos de socorro entre os grupos DU e DM. (15,8 vs. $5,3 \%, P=0,60)$

Não foram relatadas falhas na adesão à farmacoterapia, interrupção de tratamentos, ou queixas de evento adverso nos grupos durante a realização do estudo. 


\section{DISCUSSÃO}

Este estudo avaliou a eficácia da analgesia na cirurgia de extração de terceiros molares inferiores inclusos, comparando dose única de ibuprofeno 1 hora antes da cirurgia versus doses múltiplas de ibuprofeno iniciadas 25 horas antes do ato cirúrgico. O modelo experimental utilizado para a presente pesquisa foi o ensaio clínico randomizado, duplo cego, cruzado, controlado. Para avaliação da dor utilizouse a escala visual analógica, a aplicação dos filamentos Semmes-Weinstein e o consumo de analgésico socorro no período pós-operatório.

Para tanto, 19 participantes aderiram ao protocolo de pesquisa, todos adultos jovens e a maioria do gênero feminino. Todos os participantes gozavam de boa saúde geral e bucal e apresentavam indicação de exodontia bilateral de terceiros molares inclusos inferiores em posição similar. Os fatores que poderiam influenciar a dor pós-operatória em cirurgia para remoção de terceiros molares como a idade, história médica, presença de pericoronarite, má higiene oral, o tipo de inclusão foram controlados através de uma anamnese direcionada e avaliações clínica e radiográfica detalhadas.

Buscou-se maior controle entre os grupos por meio da padronização da técnica cirúrgica empregada, sendo o tempo cirúrgico e quantidade do anestésico semelhantes entre os mesmos.

A classificação da inclusão dos dentes retidos segundo Winter (1926) e Pell\&Gregory (1937), realizada na avaliação inicial permitiu a seleção de participantes que apresentavam terceiros molares retidos de forma semelhante dos dois lados, possibilitando, uma menor variabilidade entre as intervenções $(39,40)$. Isto foi confirmado por meio do tempo semelhante para execução de cada cirurgia.

O principal resultado deste estudo foi a redução significativa na EVA nas primeiras 3 e 8 horas após a cirurgia no grupo que recebeu doses múltiplas de ibuprofeno em comparação com o grupo que recebeu de dose única, o que significou clinicamente a diminuição do nível de intensidade da dor, que passou de moderada para fraca.

$\mathrm{Na}$ escala de dor, segundo a OMS (50), os valores 1 a 3 indicam a intensidade da dor fraca e de 4 a 6 indicam a intensidade de dor moderada. Observou-se que no grupo que recebeu doses múltiplas de ibuprofeno no préoperatório a dor no pós-operatório estava presente em baixa intensidade em 3 e 8 
horas, diferentes do grupo que recebeu dose única, que se manteve a um nível moderado. O pico da dor após a cirurgia de extração de terceiro molar incluso ocorre em 3 a 8 horas após o procedimento e diminui gradualmente até o segundo dia pósoperatório, quando não há complicações $(36,52)$.

A literatura cita vários métodos para avaliar a intensidade de dor, como as escalas EVA, EVN, filamentos de Semmes-Weinstein e consumo do analgésico socorro $(43,44,46,47,48)$.

A EVA é a ferramenta validada mais utilizada em pesquisas para mensurar a intensidade da sensação dolorosa. Silva e Deliberato (38) concluíram em seus estudos de revisão de artigos científicos que a EVA é um método confiável, simples e de fácil compreensão pelo paciente (38).

No presente estudo a utilização desta escala mostrou-se capaz de captar diferença significativa da intensidade da dor, além de ser confirmada a fácil compreensão e uso pelos participantes. Desta forma, pode-se afirmar que a adesão de quase todos os participantes ao protocolo foi facilitada.

A analgesia preemptiva refere-se à administração de analgésicos antes da incisão cirúrgica ou da injúria tecidual (28), e o seu objetivo é prevenir a sensibilidade dolorosa no período pós-operatório no auge da resposta, o que foi demonstrado em nosso estudo por meio da utilização de doses múltiplas préoperatórias pela via oral.

Embora sejam posologias rotineiramente usadas para extração de terceiros molares inferiores, são escassos os trabalhos comparando as duas formas de utilização, ou seja, dose única e doses múltiplas prescritas antes da cirurgia.

A extração de terceiros molares inclusos induz à dor de intensidade moderada a severa e é um modelo de dor inflamatória bastante utilizado em ensaios clínicos para estudo de analgésicos (52).

Anti-inflamatórios não esteroidais têm sido utilizados em grande número de estudos com administrações pré e pós-operatória para o alívio da dor pósoperatória. Entretanto, faltam estudos para definir o esquema terapêutico mais efetivo. Alguns estudos conseguiram demonstrar maior redução da intensidade da dor no período pós-operatório com a utilização de dose única oral pré-operatória de anti-inflamatório comparada ao tratamento pós-operatório $(5,9,10,32)$. Outros chegaram a resultados contrários, evidenciando igual ou maior benefício do tratamento oral pós-operatório em relação ao pré-operatório, quando ambos foram 
comparados entre si e a nenhum tratamento com anti-inflamatório $(10,11)$. Entretanto, quando a via endovenosa foi utilizada, houve maior redução da intensidade da dor com o pré-tratamento em dose única em relação ao póstratamento com anti-inflamatório, demonstrando eficácia da analgesia preemptiva $(6,7,8)$.

A maioria dos estudos sobre analgesia preemptiva frente à cirurgias odontológicas, utilizou dose única pré-operatória. O único estudo a comparar o efeito preemptivo de doses múltiplas pré-operatórias foi o ensaio clínico controlado por placebo, conduzido por Liporaci (13). Neste estudo, não houve superioridade do tratamento pré-operatório com doses múltiplas de cetoprofeno, realizado por 2 dias antes da cirurgia de extração de terceiro molar incluso. Entretanto, neste ensaio, o cetoprofeno continuou a ser utilizado no período pós-operatório, por 3 dias, o que pode ter mascarado resultados encontrados.

No presente estudo, utilizou-se o paracetamol no período pós-operatório nos dois grupos, e houve descontinuação do ibuprofeno para que o efeito preemptivo do anti-inflamatório pudesse ser verificado sem qualquer viés, uma vez que o mecanismo de ação do paracetamol é diferente do ibuprofeno.

A falta de evidência do benefício da administração pré-operatória em estudos clínicos pode ser explicada pela utilização de esquema de medicamento único, ao contrário da abordagem analgésica multimodal e pela utilização de dose única préoperatória, o que resulta em duração inadequada da analgesia, levando à sensibilização periférica e central após o término do efeito do medicamento (28). Considerando que o ibuprofeno leva cerca de 1 a 2 horas para ser absorvido pela via oral, e que a sua meia-vida de eliminação gira em torno de 1.8 a 2.44 horas, pressupõe-se que a administração de dose única uma hora antes da cirurgia, não apresente duração de efeito suficiente para se alcançar a analgesia preemptiva. $\mathrm{Na}$ literatura não foram encontrados estudos acerca da utilização de doses múltiplas pré-operatórias do ibuprofeno comparadas com dose única pré-operatória.

O ibuprofeno foi escolhido por apresentar menor risco de agressão gástrica, hepática e renal em relação aos outros anti-inflamatórios não esteroidais, com eficácia analgésica comparável aos mesmos, de acordo com estudos epidemiológicos (14).

Não foram observados, em ambos os grupos, problemas relacionados à segurança ou relatos de eventos adversos pelo uso dos protocolos estudados. 
Com relação à utilização de analgésico de socorro, não houve diferença significativa entre os grupos, embora mais participantes do grupo dose única, tenham utilizado

Considerando as limitações do estudo, ressaltamos que em função de razões logísticas, as avaliações com os filamentos de Semmes-Weinstein e a mensuração do grau de abertura bucal foram realizadas somente 24 horas após a cirurgia, momento em que já não havia mais diferenças detectáveis pela EVA.

Recomenda-se que em próximos estudos todas as verificações algesimétricas sejam realizadas com menor intervalo de tempo nas primeiras 24 horas pósoperatórias, que é quando ocorre o auge da resposta inflamatória. Além disto, associar um estudo farmacocinético em condições controladas, para comparar o nível plasmático do fármaco à resposta analgésica e para confirmar a utilização do analgésico (concentração e posologia). 


\section{CONCLUSÃO}

Por meio da aplicação da escala analógica visual, observou-se que o uso de doses múltiplas de ibuprofeno no período pré-operatório foi mais eficaz no controle da dor pós-operatória em cirurgia de remoção de terceiros molares inferiores inclusos em relação ao uso de dose única pré-operatória.

A utilização de filamentos Semmes-Weinstein para avaliação da dor e a medição do grau de abertura bucal não mostraram diferenças significativas referentes entre os grupos estudados.

Nenhum evento adverso foi relatado pelos participantes durante a realização deste estudo. 


\section{REFERÊNCIAS}

1 Kissin I. Preemptive analgesia.Anesthesiology.2000;93:1138-43.

2 Woolf CJ, Chong MS. Preemptive analgesia: treating postoperative pain by preventing the establishment of central sensitization. AnaesthAnalg.1993;77(2):36279.

3 Rocha APC, Kraychete DC, Lemonica L, Carvalho LRD, Barros GAMD, Garcia JBDS, Sakata RK. Dor: aspectos atuais da sensibilização periférica e central. RevBrasAnestesiol.2007; 57(1): 94-105.

4 Pimenta CAM, Santos EMM, Chaves LD, Martins LM, Gutierrez BAO. Controle da dor no pós-operatório. Rev. Esc. Enferm.USP. 2001;35(2):180-183.

5 Fletcher MC,SperaJF.Management of Acute Postoperative Pain after Oral Surgery. Dent Clin N Am. 2012; 56: 95-111.

6 Çağıran E, Eyigör C, Sezer B, Uyar M. Preemptive analgesic efficacy of dexketoprofentrometamol on impacted third molar surgery. Agri. 2014;26(1):29-33.

7 Zor ZF,Isik B, Cetiner S. Efficacy of preemptive lornoxicam on postoperative analgesia after surgical removal of mandibular third molars. Oral Surg Oral Med Oral Pathol Oral Radiol. 2014;117(1):27-3.

8 Gutta R, Koehn CR, James LE. Does ketorolac have a preemptive analgesic effect? A randomized, double-blind, control study. J Oral Maxillofac Surg. 2013 Dec; 71(12):2029-2034.

9 Aznar-Arasa L, Harutunian K, Figueiredo R, Valmaseda-Castello'n E, Gay-Escoda C. Effect of preoperative ibuprofen on pain and swelling after lowerthird molar removal: a randomized controlled trial. Int J Oral Maxillofac Surg. 2012; 41: 10051009. 
10 Kaczmarzyk T, Wichlinski J, Stypulkowska J, Zaleska M, Woron J. Preemptive effect of ketoprofen on postoperative pain following third molar surgery. A prospective, randomized, double-blinded clinical trial.Int J Oral MaxillofacSurg.2010; 39: 647-652.

11 Jung YS, Kim MK, Um YJ, Park HS, Lee EW, Kang JW.The effects on postoperative oral surgery pain by varying NSAID administration times: comparison on effect of preemptive analgesia. OralSurgOralMedOralPatholOralRadiolEndod. 2005; 100(5): 559-563.

12 Liporaci JLJ Jr. Avaliação da eficácia da analgesia preemptiva na cirurgia de extração de terceiros molares inclusos. RevBrasAnestesiol. 2012 jul-ago; 62(4): 50610.

13 Liporaci JLJ Jr. Avaliação da Eficácia da Analgesia Preemptiva na Cirurgia de Extração de Terceiros Molares Inclusos [Tese]. RibeirãoPreto: USP; 2011.

14 Rainsford, KD. Ibuprofen: pharmacology, efficacy and safety.Inflammopharmacol 2009; 17:275-342.

15 LoeserJD,Treede RD. The Kyoto protocolof IASP Basic PainTerminology.Pain. 2008;137: 473-477.

16

APED.

$[E m$

linha].

Disponível

em

<http://www.apeddor.com/index.php?lop=conteudo\&op=d67d8ab4f4c10bf22aa353e2 7879133c>[Consultado em 15/01/2015].

17 Garcia JBS, Issy AM, Sakata RK. Analgesia Preemptiva. RevBrasAnestesiol. 2001; 51(5): 448-463.

18 Kim K, Brar P, Jakubowski J, Kaltman S, Lopes E. The use of corticosteroids and nonesteroidalantiinflamatory medication for the management of pain and inflamation after third molar surgery: A review of the literature. Oral Surg Oral Med Oral Pathol Oral RadiolEndod. 2009; 107(5): 630-40. 
19 Silva JCL. Avaliação de dois protocolos farmacológicos em exodontias de terceiros molares inferiores retidos. [Tese]. Piracicaba: Faculdade de Odontologia de Piracicaba; 2011.

$20 \mathrm{Fu}$ JY, Masferrer JL, Seibert K, Raz A, Needleman P. The induction and suppression of prostaglandin $\mathrm{H} 2$ synthase (cyclooxygenase) in human monocytes.Journal of Biological Chemistry.1990;265(28): 16737-16740.

21 Fattah CMRS, AranegaAM,Leal CR, Martinho J, Costa AR. Controle da dor pósoperatória em cirurgia bucal: revisão de literatura. Rev. Odontol. Araçatuba. 2005Jul/Dez; 26(2): 56-62.

22 Wannmacher L. Antiinflamatórios não-esteróides. In Wannmacher L, Ferreira MBC.Farmacologia clínica para dentistas. 3ae edição. Rio de Janeiro: Guanabara Koogan; 2007. p. 254-269.

23 Woolf CJ. Somatic pain - pathogenesis and prevention. $\mathrm{Br} J$ Anaesth.1995; 75:169-176.

24 Wassef MR. Concepts of preemptive analgesia for postoperative pain. Mt Sinai J Med. 1998; 65(4): 271-9.

25 Rainsford, K. D. Ibuprofen: from invention to an OTC therapeutic mainstay. InternationalJournalofClinicalPractice. 2013;67(s178): 9-20.

26 Brasil. Ministério da Saúde. Secretaria de Ciência, Tecnologia e Insumos Estratégicos. Departamento de Assistência Farmacêutica e Insumos Estratégicos. Relação Nacional de Medicamentos Essenciais: Rename 2013, 8a ed.

27 Baptista RP, Zangerônimo MG, Sousa RV, Marques LS, Pereira LJ. Preemptive Analgesia in dentistry - LiteratureReview.Revista da Universidade Vale do Rio Verde. 2011; 9(1), 38-51. doi: http://dx. doi. org/10.5892/RUVRV. 91.3851. 
28 Rosero EB, Joshi GP. Preemptive, Preventive, Multimodal Analgesia: What Do They Really Mean? PlastReconstrSurg. 2014;134:85S-93S.

29 Kelly D J, Ahmad M, Brull SJ. Preemptive analgesia I: physiological pathways and pharmacological modalities. Canadian journal of anaesthesia, 2001; 48(10): 1000-1010.

30 Ong CKS, Lirk P, Seymour,RA, Jenkins BJ. The efficacy of preemptive analgesia for acute postoperative pain management: a meta-analysis. Anesthesia\& Analgesia. 2005; 100(3): 757-773.

31 Jackson DL, Moore PA, Hargreaves KM. Preoperative nonsteroidal antiinflammatory medication for the prevention of postoperative dental pain.JADA. 1989 nov; 119:641-7.

32 Savage MG, Henry MA. Preoperative nonsteroidal anti-inflammatory agents: review of the literature. Oral Surg Oral Med Oral Pathol Oral RadiolEndod.2004; 98(2):146-52.

33 Al-Sukhun J, Al-Sukhun S, Penttila H, Ashammakhi N, Al-Sukhun R. Preemptive Analgesic Effect of Low Doses of Celecoxib Is Superior to Low Doses of Traditional Nonsteroidal Anti-Inflammatory Drugs. J CraniofacSurg. 2012; 23: 526-529.

34 Wannmacher L, Ferreira MBC. Farmacologia clínica para dentistas. 3a edição. Rio de Janeiro: Guanabara Koogan; 2007. Capítulo 8, Processos farmacocinéticos e suas aplicações na prescrição, p. 68-79.

35 Boulox GF, Steed MB, Perciaccante VJ. Complications of third molar surgery. Oral MaxillofacSurgClin North Am. 2007; 19(1): 117-28.

36 Paulesini Junior W, Caixeta Neto LS, Leporace AA, Rapoporat A. Complicações associadas à cirurgia de terceiros molares: revisão de literatura. Rev. odontol. Univ. Cid. São Paulo. 2008 maio-ago; 20(2): 181-5. 
37 Arantes VMN. Analgesia preemptiva do cetoprofeno e do parecoxibe em cirurgia para remoção de terceiros molares inclusos [tese]. São Paulo: Faculdade de Medicina, Universidade de São Paulo; 2006.

38 Silva FCda, Deliberato PCP. Análise das escalas de dor: revisão de literatura. Revista Brasileira de Ciências da Saúde, São Caetano do Sul. 2009 jan/mar;19: 869.

39 Peterson LJ. Princípios de Exodontia Complicada. In: Peterson LJ. Cirurgia Oral e Maxilofacial Contemporânea. São Paulo: 2000. p. 179-213.

40 Aguiar ASWD, Oliveira ACX, Martins PC, Freire ROM. Avaliação do grau de abertura bucal e dor pós-operatória após a remoção de terceiros molares inferiores retidos. Rev Cir TraumatolBuco-Maxilo-Fac. 2005; 5(3): 57-64.

41 Winter GB. Principles of exodontia as applied to the impacted mandibular third molar. St. Louis, Mo. : American Medical Book Co. 1926; 835p.

42 Pell GS, Gregori GT.A classification of impacted mandibular third molar. J Dent Educ. 1937; 1: 157.

43 Hjermstad MJ et al. Studies comparing Numerical Rating Scales, Verbal Rating Scales, and Visual Analogue Scales for assessment of pain intensity in adults: a systematic literature review. J PainSymptom Manage. 2011; 41(6): 1073-1093.

44 Ferreira-Valente MA, Pais-Ribeiro JL, Jensen MP. Validity of four pain intensity rating scales.Pain. 2011 Oct; 152(10):2399-404.

45 Valadão CAA, Mazzei S, Oleskovicz N. Evaluation of epidural morphine or ketamine analgesic effect in dogs by von Frey filaments.ArquivoBrasileiro de MedicinaVeterinária e Zootecnia.2002; 54(4): 383-389. 
46 Weinstein S. Fifty years of somatosensory research: from the Semmes-Weinstein monofilaments to the Weinstein Enhanced Sensory Test. J Hand Ther. 1993; 6(1): 11-22.

47 Fruhstorfer H, Gross W, Selbmann O. Von Frey hairs: new materials for a new design. Eur J Pain. 2001;5(3): 341-342.

48 Lambert GA, Mallos G, Zagami AS. Von Frey's hairs-a review of their technology and use-a novel automated von Frey device for improved testing for hyperalgesia. J NeurosciMethods. 2009;177(2): 420-426.

49 Saund DS, Pearson D, Dietrich T. Reliability and validity of self-assessment of mouth opening: a validation study. BMC Oral Health.2012; 12:48.

50 World Health Organization.Traitement de la douleurcancéreuse.Geneva,Switz: World Health Organization; 1997.

51http://www.anvisa.gov.br/hotsite/notivisa/manual/ea_medicamento_profissional.pdf

52 Seymour R, Meechan JG, Blair GS. An investigation into post-operative pain after third molar surgery under local analgesia.Br J Oral MaxillofacSurg. 1985; 23: 410-8.

53 Puertas EB. Ensaio clínico randomizado, duplo-cego, comparativo entre a associação de cafeína, carisoprodol, diclofenaco sódico e paracetamol e a ciclobenzaprina, para avaliação da eficácia e segurança no tratamento de pacientes com lombalgia e lombociatalgia agudas. Acta Ortop Bras, . 2006; 14(1), 11. 


\section{APÊNDICE A -TERMO DE CONSENTIMENTO LIVRE E ESCLARECIDO - TCLE}

O (a) Senhor(a) está sendo convidado(a) a

participar do projeto: AVALIAÇÃO DO USO PREEMPTIVO DE ANTIINFLAMATÓRIO NÃO ESTEROIDAL SOBRE A HIPERALGESIA PÓS EXODONTIA DE TERCEIROS MOLARES INCLUSOS.

O objetivo desta pesquisa é avaliar se o uso de várias dosesde um medicamento para dor é melhor do que uma única dose, antes da extração do dente do siso para o controle da dor depois da extração.

$\mathrm{O}$ (a) senhor(a) receberá todos os esclarecimentos necessários antes e durante a pesquisa. Seu nome não aparecerá, sendo mantido o mais rigoroso sigilo através da omissão total de quaisquer informações que permitam identificá-lo(a).

A sua participação será por meio da realização da extração do dente do siso incluso (dente que se encontra dentro do osso da mandíbula), passando por exame odontológico antes e depois do procedimento. Antes da extração de cada um de seus dentes, o senhor(a) receberá 5 cápsulas. Em uma fase da pesquisa, todos as cápsulas serão de um remédio para dor. Em outra fase, apenas um desses cápsula será de um remédio para a dor, sendo os outros três compostos por uma substância sem efeito(placebo). O(a) senhor(a) não saberá qual dessas cápsulas tem medicamento e qual não tem. Depois da extração, o(a) senhor(a) também receberá doses de outros medicamentos para a dor. A avaliação após a extração do dente será por meio da aplicação de finos filamentos de plástico dobrável na região que foi operada, e medida da abertura da boca no dia seguinte. Além disso, o senhor(a) deverá responder a uma escala de dor nos horários solicitados pelo pesquisador e medir o número de comprimidos dos medicamentos para a dor utilizados depois da cirurgia. Os exames e a extração do dente ocorrerão na Clínica Odontológica da Universidade de Brasília. Os exames durarão cerca de 20 minutos, enquanto a extração poderá durar até 2 horas. Após a cirurgia, 0 senhor(a) poderá ter dor e inchaço da região operada. Os riscos incluem infecção no local da cirurgia, fratura da raiz do dente, perda da sensibilidade temporária no lado operado, dificuldade de abertura de boca. Os remédios utilizados poderão dar dor de estômago, problemas no fígado (se utilizados de maneira incorreta) e sonolência e moleza. Os benefícios incluem a retirada dos dentes do siso inclusos, prevenindo da ocorrência de cistos dos maxilares e 
prevenindo também o nascimento dos dentes do siso em posição errada. Além disso, os remédios utilizados poderão diminuir a dor após a extração do dente.

O pesquisador deste projeto se compromete em dar o conhecimento ao paciente de todos os eventos adversos ocorridos durante o desenvolvimento desta pesquisa.

Informamos que o(a) Senhor(a) pode se recusar a participar de qualquer procedimento, qualquer questão que lhe traga constrangimento, podendo desistir de participar da pesquisa em qualquer momento sem nenhum prejuízo para o(a) senhor(a). Sua participação é voluntária, isto é, não há pagamento por sua colaboração.

Os resultados da pesquisa serão divulgados na Universidade de Brasília podendo ser publicados posteriormente. Os dados e materiais utilizados na pesquisa ficarão sob a guarda do pesquisador por um período de no mínimo cinco anos, após isso serão destruídos ou mantidos na instituição.

Se o(a) Senhor(a) tiver qualquer dúvida em relação à pesquisa, por favor telefone para: Dr(a). Sara Katerine, da Universidade de Brasília, telefone: 91544316, no horário comercial.

Este projeto foi Aprovado pelo Comitê de Ética em Pesquisa da Faculdade de Ciências da Saúde da Universidade de Brasília. As dúvidas com relação à assinatura do TCLE ou os direitos do participante da pesquisa podem ser obtidos através do telefone: (61) 3107-1947.

Este documento foi elaborado em duas vias, uma ficará com o pesquisador responsável e a outra com o participante da pesquisa.

Brasília, de de 


\section{APÊNDICE B - ORIENTAÇÕES PÓS-OPERATÓRIAS}

PACIENTE PRONTUÁRIO

Cuidados após a cirurgia:

No primeiro dia:

- Aplicar gelo no local por 10 minutos e descansar por 60 minutos, (coloque o gelo em saco plástico e proteja a pele com tecido fino).

- Repouso físico: evite caminhadas, ginástica e esportes de contato. Evitar conversas longas.

- $\quad$ Após as refeições escovar e lavar cuidadosamente a boca.

- Não fazer bochechos. Na escovação dentária apenas enxaguar a boca delicadamente e não cuspir.

- Evitar sol e altas temperaturas.

- Alimentação líquida ou pastosa e fria (Sorvete, Sucos, Cremes, logurte, Gelatinas, Sopa passada no liquidificador e purês).

- Dormir com 2 travesseiros (cabeça mais alta que o corpo).

- Caso saia um pouco de sangue, morda ou faça compressão de gaze no local por 30 minutos. Repetir a manobra em caso de necessidade.

Segundo dia:

- Alimentação leve.

- Evitar exercícios físicos aeróbicos e exposição do sol até remoção dos pontos.

- Após a escovação dentária, realizar bochecho cuidadoso com clorexidina $0,12 \%$ (Periogard $\AA$ ), pela manhã e pela noite.

Observações: tome o medicamento prescrito no dia e hora corretos. Anote sua avaliação da dor na ficha nos horários marcados.

$1^{\circ}$. Retorno:

Data para remoção de pontos: 


\section{ANEXO 1 - APROVAÇÃO COMITÊ DE ÉTICA}

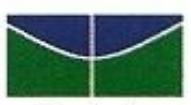

Universidade de Brasilia

Faculdade de Ciências da Saúde

Comitê de Ética em Pesquisa - CEP/FS

\section{PROCESSO DE ANÁLISE DE PROJETO DE PESQUISA}

Título do Projeto: "Avaliaçāo do Uso Preemptivo de Antiinflamatório não Esteroidal sobre a Hiperalgesia pós Exodontia de Terceiros Molares Inclusos"

Pesquisadora Responsável: Sara Katerine Vieira

Data de Entrada: 07/02/2014

CAAE: 14533413.3 .0000 .0030

Com base na Resolução 466/12, do CNS/MS, que regulamenta a ética em pesquisa com seres humanos, o Comitê de Ética em Pesquisa com Seres Humanos da Faculdade de Ciências da Saúde da Universidade de Brasília, após análise dos aspectos éticos e do contexto técnico-científico, resolveu APROVAR o projeto intitulado "Avaliação do Uso Preemptivo de Antiinflamatório não Esteroidal sobre a Hiperalgesia pós Exodontia de Terceiros Molares Inclusos”, na $1^{\mathrm{a}}$ reunião ordinária realizada no dia 12 de março de 2014.

O pesquisador (a) responsável fica, desde já, notificado (a) da obrigatoriedade da apresentação de um relatório semestral e relatório final sucinto e objetivo sobre o desenvolvimento do Projeto, no prazo de 1 (um) ano a contar da presente data presente.

Brasília, 13 de maio de 2014.
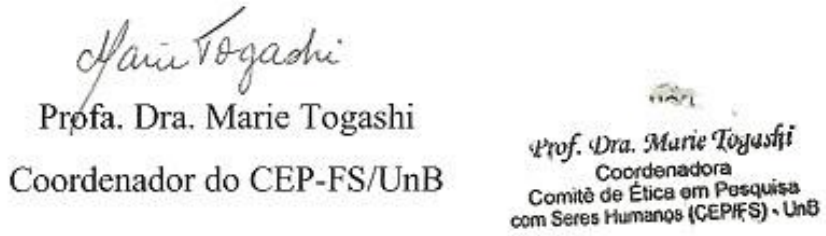

Comitê de Ética em Pesquisa com Seres Humanos - Faculdade de Ciências da Saúde

Universidade de Brasilia - Campus Universitário Darcy Ribeiro - CEP: 70.910-900 Telefone: (61)-3107-1947 Email: cepfs@unb.br 